\title{
Finitely presented dynamical systems
}

\author{
DAVID FRIED \\ Department of Mathematics, Boston University, 111 Cummington Street, Boston, MA \\ $02215, \boldsymbol{U S A}$
}

(Received 1 October 1986 and revised 12 January 1987)

Abstract We extend results of Bowen and Manning on systems with good symbolic dynamics In particular we identify the class of dynamical systems that admit Markov partitions For these systems the Manning-Bowen method of countıng periodic points is explained in terms of topological coincidence numbers We show, in particular, that an expansive system with a finite cover by rectangles has a rational zeta function

We first recall some of the theory of expansive homeomorphisms, as presented for instance in [DGS] Let $\Omega$ be a compact topological space, $f \Omega \rightarrow \Omega$ a homeomorphism We say $f$ is expansive if there is a closed neighbourhood $V \subset \Omega \times \Omega$ of the diagonal $\Delta_{\Omega}$ such that $F=f \times f \Omega \times \Omega \rightarrow \Omega \times \Omega$ satisfies

$$
\bigcap_{i \in \mathbf{Z}} F^{-1} V=\Delta_{\Omega}
$$

One calls $V$ an expansive index for $f$ We will see that $f$ expansive $\Rightarrow \Omega$ metrizable (Lemma 2) In terms of a metric $d$ on $\Omega$ this means that there is an expansive constant $c>0$ such that

$$
p, q \in \Omega, p \neq q \Rightarrow \text { for some } \imath \in \mathbb{Z}, d\left(f^{\prime} p, f^{\prime} q\right)>c
$$

For $\varepsilon>0$ and $x \in \Omega$ define the $\varepsilon$-stable set to be

$$
W_{\varepsilon}^{s}(x)=\left\{y \mid d\left(f^{\prime} x, f^{\prime} y\right) \leq \varepsilon \text { for } \imath \geq 0\right\}
$$

and the $\varepsilon$-unstable set to be

$$
W_{\varepsilon}^{u}(x)=\left\{y \mid d\left(f^{\prime} x, f^{\prime} y\right) \leq \varepsilon \text { for } \imath \leq 0\right\}
$$

If $\varepsilon \leq c / 2$ then for any $x, y \in \Omega$ the intersection of $W_{\varepsilon}^{s}(x)$ with $W_{\varepsilon}^{u}(y)$ consists of at most one point Let

$$
D_{\varepsilon}=\left\{(x, y) \in \Omega \times \Omega \mid W_{\varepsilon}^{s}(x) \text { meets } W_{\varepsilon}^{u}(y)\right\}
$$

and define $[,] D_{\varepsilon} \rightarrow \Omega$ so that

$$
[x, y] \in W_{\varepsilon}^{s}(x) \cap W_{\varepsilon}^{u}(y)
$$

Then $D_{\varepsilon}$ is closed in $\Omega \times \Omega$ and [ , ] is continuous, as is easily checked We say $R \subset \Omega$ is a rectangle if $R \times R \subset D_{\varepsilon}$ Then if $x \in R$ one has the sets

$$
W_{\varepsilon}^{s}(x, R)=R \cap W_{\varepsilon}^{s}(x), \quad W_{\varepsilon}^{u}(x, R)=R \cap W_{\varepsilon}^{u}(x)
$$


and a natural homeomorphism (justıfyıng the name rectangle)

$$
R \simeq W_{\varepsilon}^{u}(x, R) \times W_{\varepsilon}^{s}(x, R)
$$

sending the point $y \in R$ to the pair $([y, x],[x, y])$ and the pair $(y, z)$ to the point $[y, z]$ If $\Omega$ is a finite union of rectangles we say $f$ is FR this is a local property of $f, 1 \mathrm{e}$ it $1 \mathrm{~s}$ enough that every $x \in \Omega$ have a neighbourhood that is a finite union of rectangles

Next we study factors of subshifts Take $\mathscr{S}$ a finite set of 'symbols' and consider the shift map $\sigma\left(s_{n}\right)=\left(s_{n+1}\right)$ on sequences $\left(s_{n}\right), n \in \mathbb{Z}, s_{n} \in \mathscr{S}$ When the sequence space $\mathscr{S}^{\mathbb{Z}}$ is endowed with the product topology it is compact and metrizable and $\sigma$ is expansive If $\Sigma \subset \mathscr{S}^{\mathbb{Z}}$ is a closed $\sigma$-invariant subset we say $\sigma \Sigma \rightarrow \Sigma$ (or $\Sigma$ itself) is a subshift with symbol set $\mathscr{S}$ If $f \Omega \rightarrow \Omega$ is a factor of $\Sigma$ by a surjective semiconjugacy $\pi \quad \Sigma \rightarrow \Omega$ then the equivalence relation $E \subset \Sigma \times \Sigma \subset \mathscr{S}^{\mathbb{Z}} \times \mathscr{S}^{\mathbb{Z}} \simeq(\mathscr{S} \times \mathscr{S})^{\mathbb{Z}}$

$$
x E y \Leftrightarrow \pi x=\pi y
$$

1s $\sigma \times \sigma$-1nvariant, so $E$ is a subshift with symbol set $\mathscr{S} \times \mathscr{S}$ A subshift $\Sigma$ is defined completely by saying which cylinders are disjoint from $\Sigma$, where a cylinder $C$ is a closed-open subset of $\mathscr{S}^{\mathbb{Z}}$ whose coordinates are specified in finitely many places If there are finitely many cylinders $C_{i}$ such that

$$
\mathscr{P}^{\mathbb{Z}}-\Sigma=\bigcup_{i} \bigcup_{n \in \mathbb{Z}} \sigma^{n} C_{t}
$$

then $\Sigma$ is a subshift of finite type (SFT) If both $\Sigma$ (as a subshift on $\mathscr{P}$ ) and $E$ (as a subshift on $\mathscr{S} \times \mathscr{P}$ ) are of finite type we say $f$ is finitely presented (FP) This is Gromov's terminology, suggested by a loose analogy with groups $\Sigma$ is analogous to the free group $F$ on a finite set, $E$ to the normal closure $R$ of a finite set, so $\Sigma / E$ 1s like the finitely presented group $F / R$

Suppose $\Sigma$ is an SFT and $\Omega$ is a factor of $\Sigma$ We will show

LEMMA $1 \Omega$ is expansive $\Leftrightarrow E$ is of fintte type

Thus if $\Omega \simeq \Sigma / E \simeq \Sigma^{\prime} / E^{\prime}$ is a factor of SFT's in two ways and $E$ is of finite type then $E^{\prime}$ is of finite type (In group theory, if $F / R \simeq F^{\prime} / R^{\prime}$, with $F, F^{\prime}$ free groups on finitely many generators and $R$ is the normal closure of a finite set then so is $R$, so Gromov's analogy holds up) Actually we will show

TheOREM $1 f i s F P \Leftrightarrow f i s F R$

so that FP systems have a local dynamical characterization and each FR system has a finite symbolic description

Following Bowen's book [B1] we define a Markov partition for an expansive homeomorphism $f \Omega \rightarrow \Omega$ to be a finite cover $\mathcal{M}$ by proper rectangles ( $R$ is proper if $R=\overline{\text { int } R}$ ) with diameter $<\varepsilon$ such that if $x \in$ int $R, f x \in$ int $R^{\prime}, R, R^{\prime} \in \mathcal{M}$, then

$$
f\left(W_{\varepsilon}^{s}(x, R)\right) \subset R^{\prime} \quad \text { and } \quad f^{-1}\left(W_{\varepsilon}^{u}\left(f x, R^{\prime}\right)\right) \subset R
$$

If $f$ has a Markov partition we say it is MP We will show

THEOREM $2 f i s F P \Leftrightarrow f i s M P$ 
Here the backward implication is essentially a remark of Bowen ([B2, p 13]) from which the notion of a finitely presented system originates Altogether we have

$$
F P \Leftrightarrow F R \Leftrightarrow M P \Leftrightarrow \text { expansive factor of SFT }
$$

The primary example of FP systems are Smale's Axiom A basic sets Here $\Omega$ is expansive and has canonical coordinates (CC) 1 e $D_{\varepsilon}$ is a neighbourhood of $\Delta_{\Omega}$ so that $[x, y]$ makes sense if $x, y \in \Omega$ are sufficiently close (this reflects the uniform transversality of the stable and unstable manifolds) Also $\Omega$ has a metric $d$ (induced from a well-chosen Riemannian metric) that contracts $\varepsilon$-stable sets and expands $\varepsilon$-unstable sets uniformly, 1 e $\exists \lambda \in(0,1)$ so that if $y \in W_{\varepsilon}^{s}(x)$ and $z \in W_{\varepsilon}^{u}(x), x, y, z \in$ $\Omega$, then

$$
d(f z, f y) \leq \lambda d(x, y), \quad d\left(f^{-1} x, f^{-1} z\right) \leq \lambda d(x, z)
$$

From (*) and CC, Bowen deduced the pseudo-orbit tracing property (shadowing), and from this he exhibited $\Omega$ as a factor of an SFT Then using this presentation and CC he obtained a Markov partition [B1] We will essentially follow this last step in our proof of the forward implication in Theorem 2

Ruelle defined a Smale space to be a compact metric space and an expansive homeomorphism, with $\mathrm{CC}$ and $(*)$ [R] He observed that these properties were enough to produce Markov partitions In fact the metric comes for free We will show

LEMMA 2 Given an expansive system $f \Omega \rightarrow \Omega$ there is a metric $d$ on $\Omega$ such that (*) holds and $f$ is a Lipschitz isomorphism The Lipschitz class of $d$ is determined by an expansive index $V$ for $f$ and the Holder class of $d$ is uniquely determined by $f$ If $f^{\prime} \Omega^{\prime} \rightarrow \Omega^{\prime}$ is another expansive system endowed with a metric $d^{\prime}$ in this natural Holder class then any semiconjugacy $\pi \Omega \rightarrow \Omega^{\prime}$ is Holder continuous

In particular there is an intrinsic notion of exponential convergence for an expansive system These natural metrics will be used in our proof of Theorem 1 For a Smale space they were constructed in [F3] in terms of a finite presentation

Our second motivating example arises when $\Omega$ is zero-dimensional Then $\Omega$ is a subshift (partition $\Omega$ into finitely many small closed-open sets) and so we obtain the class of subshifts that are factors of SFT's These are just Weiss's sofic systems ([We], [CP]) It is known that a sofic $\Omega$ has a finite-to-one extension $\pi \quad \Sigma \rightarrow \Omega$ with $\Sigma$ an SFT that is s-resolving, 1 e $\pi$ is $1-1$ on each $W_{\varepsilon}^{s}(x)$ For an FR $\Omega$ we will show the corresponding result

LEMMA $3 \Omega$ has a finte-to-one s-resolving extension $G \rightarrow \Omega$ such that $G$ has $C C$

This is also used in Theorem 1

The third example of FP systems in the literature is Thurston's pseudo-Anosov homeomorphisms These obviously satisfy FR For them Markov partitions were first constructed by direct means [FLP] Our method amounts to DAing, taking the Markov partition for the resultıng hyperbolic repeller, and collapsing back down to a Markov partition for $\Omega$ itself, except that we work externally the repeller is replaced by the s-resolution of Lemma 3 
The results discussed so far are proven in $\S 1$ In $\S 2$ we turn to counting periodic points The zeta function of an expansive $f \Omega \rightarrow \Omega$

$$
\begin{gathered}
\zeta_{f}(t)=\exp \sum_{p>0} N_{p} \frac{t^{p}}{p}, \\
N_{p}=\left|\left\{x \mid f^{p} x=x\right\}\right|,
\end{gathered}
$$

is a generating function for the sequence of numbers of periodic points of various periods We will go over the Mannıng-Bowen argument for $f$ FP to express $N_{p}$ in terms of traces of certain linear maps $([\mathbf{M}],[\mathbf{B 2}])$ and we take a step towards comparing this method to the enumeration of $N_{p}$ for a basic set $\Omega$ via the Lefschetz fixed point theorem ([G1], [F2]) Namely the traces give the coincidence number of a certain simplicial correspondence depending on the presentation $\Omega=\Sigma / E$, this coincidence number counts the components in the coincidence set of this correspondence and each component corresponds to a unique fixed point of $f^{p}$ (see Theorem 3) This partially settles a question of Bowen ([B2, pp 14-15]) of how Manning's work was related to Lefschetz theory (see also [R, problem B 9]) The end result is a formula for $\zeta_{f}(t)$ that shows it is rational in $t$

This paper is intended to add some steps to Bowen's program of using symbolic dynamics to study basic sets ([B2, pp 10-15]) and to integrate the three FP examples cited above into one theory One can view FP systems as higher dimensional sofic systems or as a generalization of Smale spaces (every point has a neighbourhood that is a finite union of rectangles)

We thank Douady, Gromov, Kitchens and Marcus for their helpful conversations and Shishikura for his result cited in $\S 2$ We are especially grateful to the late Rufus Bowen for his stimulation and encouragement

The author was partially supported by the National Science Foundation, the Sloan Foundation, the IHES, and the University of Warwick

\section{Proofs of Theorems 1 and 2}

We begin by deducing Theorem 1 from the above lemmas Suppose $\Omega$ is FP with a surjective semiconjugacy $\pi \quad \Sigma \rightarrow \Omega$ Lemma 1 shows $\Omega$ is expansive Partition $\Sigma$ into cylinder sets $C$ by specifying the coordinates of a sequence between $-n$ and $n$, $n$ large Then each $C$ is a rectangle since $\Sigma$ has finite type If $n$ is large enough then $\pi(C)$ will be a small rectangle The cover $\{\pi(C)\}$ shows $f$ is FR

Conversely suppose $\Omega$ is FR Lemma 3 gives an extension $X$ with CC Give $X$ the metric of Lemma 2 Then $X$ is a Smale space, hence Bowen's arguments give shadowing and a finite presentation for $X$ So $\Omega$ is a factor of an SFT, so Lemma 1 shows $\Omega$ is FP

To prove Lemma 1 , recall that an invariant set $I$ for a homeomorphism $h$ is isolated if $I$ is the largest invariant set in some open set $U_{1}$ e if $I=\bigcap_{i \in \mathbb{Z}} h^{-1} U$ One easily shows

(A) $f \Omega \rightarrow \Omega$ is expansive $\Leftrightarrow \Delta_{\Omega} \subset \Omega \times \Omega$ is isolated for $h=f \times f \Omega \times \Omega \rightarrow \Omega \times \Omega$ This is the definition of expansive with $U$ an expansive index

(B) A subshift $\Sigma \subset \mathscr{S}^{\mathbb{Z}}$ is isolated for the full shift $h=\sigma$ on $\mathscr{S}^{\mathbb{Z}} \Leftrightarrow \Sigma$ is an SFT 
This is the definition of SFT together with the fact that unions of cylinders form a neighbourhood basis for $\mathscr{S}^{\mathbb{Z}}$

(C) Given a surjective semiconjugacy $\rho \quad X \rightarrow Y$ of homeomorphisms of compact Hausdorff spaces and an invariant set $I \subset Y, I$ is isolated $\Leftrightarrow \rho^{-1} I$ is 1solated This uses the definition of isolated and the fact that $\rho^{-1} I$ is the intersection of sets $\rho^{-1} \mathcal{O}, \mathcal{O}$ open in $Y$ Now apply (C) to $\rho=\pi \times \pi \quad \Sigma \times \Sigma \rightarrow \Omega \times \Omega$ with $I=\Delta_{\Omega}$ and use (A) and (B) Lemma 1 follows

Now we prove Lemma 2 Let $V_{n}=\bigcap_{i=-n}^{n} F^{-1} V$ for $n \geq 0$, with $F=f \times f$ Then each $V_{n}$ is a neighbourhood of $\Delta_{\Omega}$ and $\cap V_{n}=\Delta_{\Omega}$ so $\Omega$ is Hausdorff Now a compact Hausdorff space has a unique uniformity consisting of all neighbourhoods of the diagonal [K] Thus if $n$ is sufficiently large $V_{n} \circ V_{n} \subset V$ We call the least $n \geq 1$ with this property the lag $n(V)$ We have $V_{n+k} \circ V_{n+k} \subset V_{k}$ for all $k \geq 0$, with $n=n(V)$ The sets $U_{m}=V_{2 m n}, m \geq 0$, satisfy the condition

$$
U_{m} \circ U_{m} \circ U_{m} \subset U_{m-1}
$$

of the Frink Metrization Lemma ([K, p 185]) and so there is a metric $d$ on $\Omega$ such that on $\Omega \times \Omega$

$$
\left\{(x, y) \mid d(x, y)<2^{-m}\right\} \subset U_{m} \subset\left\{(x, y) \mid d(x, y)<2^{1-m}\right\}
$$

Now take $f^{\prime} \Omega^{\prime} \rightarrow \Omega^{\prime}$ expansive and in the same way construct $d^{\prime}$ from an expansive index $V^{\prime}$ with some lag $n^{\prime}$ Then to any continuous semiconjugacy $\pi \Omega \rightarrow \Omega^{\prime}$ there 1s a $\mu$ such that $\rho=\pi \times \pi \Omega \times \Omega \rightarrow \Omega^{\prime} \times \Omega^{\prime}$ satisfies $\rho V_{\mu} \subset V^{\prime}$ Then $\rho V_{\mu+1} \subset V_{1}^{\prime}$ for all $\imath \geq 0$ and so $\rho U_{m} \subset U_{m}^{\prime}$ if $2 m^{\prime} n^{\prime} \leq 2 m n-\mu$ Given $(x, y) \in V, x \neq y$, we take $a \geq 0$ so that $m=a n^{\prime}$ satisfies $2^{-m-n^{\prime}} \leq d(x, y)<2^{-m}$ Then $(x, y) \in U_{m}$ and $\rho(x, y) \in U_{m}^{\prime}$ with $m^{\prime}=a n-n_{0}$, with $n_{0} \in \mathbb{Z}^{+}$depending only on $\mu$ and $n^{\prime}$ Thus

$$
d^{\prime}(\pi x, \pi y)<2^{1-m^{\prime}} \leq c d(x, y)^{n / n^{\prime}},
$$

where $c$ depends only on $\mu, n$ and $n^{\prime}$ Thus $\pi$ is Holder with exponent $n / n^{\prime}$ the ratio of lags for $V$ and $V^{\prime}$

As a special case suppose $\Omega^{\prime}=\Omega, V^{\prime}=V, \pi=1 \mathrm{~d}$ We see that 2 metrics $d, d^{\prime}$ satısfying (F) are Lipschitz equivalent, as $n / n^{\prime}=1$ If $\Omega^{\prime}=\Omega, V^{\prime}=V, \pi=f^{ \pm 1}$ we see likewise that $f^{ \pm 1}$ are Lipschitz Finally changing $V$ leaves the Holder class of $d$ unchanged

Next take $(x, y) \in V$ such that $y \in W_{1}^{s}(x)$ Then $(x, y) \in \bigcap_{-\infty}^{0} F^{\prime} V$ If $d(x, y)<2^{-m}$ then $(x, y) \in \bigcap_{-\infty}^{2 m n} F^{\prime} V$ Thus if $a \in \mathbb{Z}^{+}$one has

$$
\left(f^{2 n a} x, f^{2 n a} y\right) \in \bigcap_{-\infty}^{2 n(m+a)} F^{\prime} V \subset U_{m+a}
$$

so that $d\left(f^{2 n a} x, f^{2 n a} y\right)<2^{1-m-a}$ Assuming that $x \neq y$ and $M$ is chosen so $d(x, y) \geq$ $2^{-1-m}$ this gives

$$
d\left(f^{2 n a} x, f^{2 n a} y\right) \leq 4\left(\frac{1}{2}\right)^{a} d(x, y)
$$

In particular the iterate $f^{6 n}$ contracts $W_{1}^{s}(x)$ by a factor $\frac{1}{2}$

Now we use an argument of Mather to alter $d$ within its Lipschitz class so that $f$ contracts these stable sets directly Take $\alpha=2^{1 / 6 n}>1$ and define

$$
e(x, y)=d(x, y)+\alpha d(f x, f y)+\quad+\alpha^{6 n-1} d\left(f^{6 n-1} x, f^{6 n-1} y\right)
$$


Then $e$ and $d$ are Lipschitz equivalent metrics and for $(x, y)$ as above

$$
\begin{aligned}
e(f x, f y) & =\alpha^{-1}(e(x, y)-d(x, y))+\alpha^{6 n-1} d\left(f^{6 n} x, f^{6 n} y\right) \\
& \leq \alpha^{-1} e(x, y)
\end{aligned}
$$

Thus (*) holds for $e$ with $\lambda=\alpha^{-1}=2^{-1 / 6 n}$ and $\varepsilon$ sufficiently small This proves Lemma 2

We now prove Lemma 3 Take the metric $d$ given in Lemma 2 and cover $\Omega$ by finitely many rectangles $R_{s}, s \in \mathscr{P}$ As $D_{\varepsilon}$ is closed, the closure of any rectangle is again a rectangle and so we may assume that each $R_{s}$ is closed Also we may suppose diam $\left(R_{s}\right)<c / 10$, where $c$ is an expansive constant To each $x \in \Omega$ we have a symbol set, a core, a star and a second star as follows

$$
\begin{aligned}
\mathscr{P}(x) & =\left\{s \in \mathscr{P} \mid x \in R_{s}\right\} \\
\operatorname{Core}(x) & =\Omega-\bigcup R_{t}, \quad t \in \mathscr{S}-\mathscr{S}(x) \\
\operatorname{Star}(x) & =\bigcup R_{s}, \quad s \in \mathscr{S}(x) \\
\operatorname{Star}_{2}(x) & =\bigcup \operatorname{Star}(y), \quad y \in \operatorname{Star}(x)
\end{aligned}
$$

Clearly $x \in \operatorname{Core}(x) \subset \operatorname{Star}(x) \subset \operatorname{Star}_{2}(x) \subset \Omega$ Core $(x)$ 1s open and $y \in \operatorname{Core}(x) \Leftrightarrow$ $\operatorname{Star}(y) \subset \operatorname{Star}(x) \Rightarrow \operatorname{Star}_{2}(y) \subset \operatorname{Star}_{2}(x)$ Choose $\delta>0$ so that disjoint rectangles $R_{s}, R_{t}, s, t \in \mathscr{Y}$, satisfy $d\left(R_{s}, R_{t}\right)>2 \delta$ and so that for any $z \in \Omega$ there is an $x \in \Omega$ with $B_{2 \delta}(z) \subset$ Core $(x)$, 1 e $2 \delta$ is a Lebesgue number for the open cover of $\Omega$ by cores The first property of $\delta$ implies that $B_{2 \delta}(z) \subset \operatorname{Star}_{2}(z)$

The relation $y \sim z \Leftrightarrow y \in W_{\varepsilon}^{u}(z)$ defines an equivalence relation $\sim$ on $\operatorname{Star}_{2}(x)$ To $y \in \operatorname{Star}_{2}(x)$ we define the projection $P(y, x) \subset \operatorname{Star}_{2}(x) / \sim$ to be the image of $W_{\varepsilon}^{s}(y) \cap$ $\operatorname{Star}_{2}(x)$ The set $\mathscr{P}(x)=\left\{P(y, x) \mid y \in \operatorname{Star}_{2} x\right\}$ is finite and indeed so is

$$
\sup _{x \in \Omega}|\mathscr{P}(x)|=K
$$

We define an $s$-germ at $x$ to be a sequence $P_{\imath} \in \mathscr{P}\left(f^{i} x\right), \imath \in \mathbb{Z}$, such that $\forall m, n \in \mathbb{Z}$ with $m \leq n, \exists y_{m n} \in \bigcap_{1=m}^{n} f^{-\imath} \operatorname{Star}_{2}\left(f^{\prime} x\right)$ with $P\left(f^{\prime} y_{m n}, f^{\prime} x\right)=P_{1}$ for $m \leq \imath \leq n$ Clearly $P_{1}=P\left(f^{i} x, f^{i} x\right)$ defines an $s$-germ (take all $y_{m n}=x$ ) that we call trivial An $s$-germ at $x$ describes a possible behaviour for the stable sets at points near $x$ Clearly $g=\left(P_{1}\right)$ is determined by its values for large $\imath$ Thus if $G_{x}$ denotes the set of $s$-germs at $x,\left|G_{x}\right| \leq K$

Let $G=\bigcup G_{x}, x \in \Omega$, be the set of all $s$-germs and let $\pi \quad G \rightarrow \Omega$ be the natural projection We see that $\pi$ is onto and has bounded fibers There is a natural bijection $h G \rightarrow G$ that lifts $f$, namely $h\left(P_{1}\right)=\left(P_{\imath+1}\right) \in G_{f x}$ for $g=\left(P_{\imath}\right) \in G_{x}$ For $z \in \Omega$ define $V(z) \subset G \times G$ so that with the relation $\sim$ on $\operatorname{Star}_{2}(z), g=\left(P_{1}\right) \in G_{x}$ and $g^{\prime}=\left(P_{\imath}^{\prime}\right) \in G_{x}$ one has $\left(g, g^{\prime}\right) \in V(z)$ if

( $\alpha) B_{\delta}(x) \cup B_{\delta}\left(x^{\prime}\right) \subset$ Core $(z)$,

( $\beta$ ) $B_{\delta}\left(x, P_{0}\right) / \sim \subset P_{0}^{\prime} / \sim$ and $B_{\delta}\left(x^{\prime}, P_{0}^{\prime}\right) / \sim \subset P_{0} / \sim$,

where $B_{\delta}(x, P), P \in \mathscr{P}(x)$, denotes the intersection of $P$ with the image of $W_{\delta}^{s}(x)$ We will regard $g, g^{\prime} \in B$ as close if $\left(g, g^{\prime}\right) \in V(z)$ for some $z$ more precisely we define $V=\bigcup_{z} V(z) \subset G \times G$ and $H=h \times h \quad G \times G \rightarrow G \times G$ and we prove that the sets $V_{n}=\bigcap_{t=-n}^{n} H^{\prime} V$ are a basis for a uniformity on $\Omega$ This defines a topology on $\Omega$ and we will see that the various properties required for Lemma 3 hold 
First we show that if $n$ is sufficiently large then $V_{n} \circ V_{n} \subset V$ Say $\left(g, g^{\prime}\right) \in V_{n}$ and $\left(g^{\prime}, g^{\prime \prime}\right) \in V_{n}$ If $n$ is large then $x=\pi g, x^{\prime}=\pi g^{\prime}$, and $x^{\prime \prime}=\pi g^{\prime \prime}$ are very close and so by our choice of $\delta$ we can find $z^{\prime} \in \Omega$ so that $B_{2 \delta}(x) \cup B_{2 \delta}\left(x^{\prime \prime}\right) \subset$ Core $\left(z^{\prime}\right)$ We also have $\left(h^{\prime} g, h^{\prime} g^{\prime}\right) \in V\left(z_{\imath}^{\prime \prime}\right),\left(h^{\prime} g^{\prime}, h^{\prime} g^{\prime \prime}\right) \in V\left(z_{\imath}\right)$ for $z_{\imath}^{\prime \prime}, z_{1} \in \Omega,|\imath| \leq n$ By symmetry, it suffices to show that in $\operatorname{Star}_{2}\left(z^{\prime}\right) / \sim$, with $g=\left(P_{1}\right), g^{\prime}=\left(P_{1}^{\prime}\right)$ and $g^{\prime \prime}=\left(P_{1}^{\prime \prime}\right)$ one has $B_{\delta}\left(x, P_{0}\right) / \sim \subset P_{0}^{\prime \prime} / \sim$ But if $w \in W_{\delta}^{s}(x)$ and $W_{\varepsilon}^{u}(w)$ meets $P_{0}$ we have in $\operatorname{Star}_{2}\left(z_{0}^{\prime \prime}\right)$ the relation $w \sim w^{\prime}, w^{\prime} \in P_{0}^{\prime}$ (use $(\beta)$ on $\left(g, g^{\prime}\right) \in V\left(z_{0}^{\prime \prime}\right)$ ) Applying $f^{n}$ gives $f^{n} w^{\prime} \in$ $B_{\delta}\left(f^{n} x^{\prime}, P_{n}^{\prime}\right)$, if $n$ is sufficiently large Then $\left(h^{n} g^{\prime}, h^{n} g^{\prime \prime}\right) \in V\left(z_{n}\right)$ implies $f^{n} w^{\prime} \sim f^{n} w^{\prime \prime}$ for some point $f^{n} w^{\prime \prime} \in P_{n}^{\prime \prime}$ Thus $w^{\prime \prime}=\left[x^{\prime \prime}, w\right]$ exists Again we assume $n$ large enough so that $d\left(x^{\prime \prime}, w^{\prime \prime}\right)<2 \delta$ (note $\left(x^{\prime \prime}, w^{\prime \prime}\right)$ is near $(x, w)$ for $x^{\prime \prime}$ close enough to $x$ ) Then, by our choice of $\delta, w^{\prime \prime}$ is an interior point of $\operatorname{Star}_{2}\left(x^{\prime \prime}\right)$ Thus $w^{\prime \prime} \in P_{0}^{\prime \prime}$ and $w \sim w^{\prime \prime}$ in $\operatorname{Star}_{2}\left(z^{\prime}\right)$, as desired

From $V_{n} \circ V_{n} \subset V$ follows $V_{n+h} \circ V_{n+k} \subset V_{k}$ for $k \geq 0$, so the $V_{n}$ are indeed a basis for a uniformity Clearly $h \quad G \rightarrow G$ is a homeomorphism

Next we show that $\Delta_{G}=\bigcap V_{k}$ Surely $(g, g) \in V$ for all $g \in G$ by our choice of $\delta$ Thus $\Delta_{G} \subset V_{k}$ for all $k$ If $\left(h^{\prime} g, h^{\prime} g^{\prime}\right) \in V$ for all $t \in \mathbb{Z}$ then $g, g^{\prime} \in G_{\curlyvee}$ for some $x \in \Omega$ since $(\pi \times \pi)(V)$ is an expansive index for $\Omega$ (this also shows $\pi$ is continuous) We have

$$
B_{\delta}\left(f^{\prime} x, P_{t}\right)=B_{\delta}\left(f^{\prime} x, P_{t}^{\prime}\right)
$$

for $g=\left(P_{1}\right), g^{\prime}=\left(P_{t}^{\prime}\right), \imath \in \mathbb{Z}$ Takıng $M \gg 0$ one sees that $P_{1-M}=P_{1-M}^{\prime}$ Thus lettıng $l \rightarrow+\infty$ we see $g=g^{\prime}$, as desired (This also shows that distınct $s$-germs at $x$ cannot be forward asymptotic, so $\pi$ is 1-1 on stable sets)

Next we show $G$ is sequentially compact Take $g^{(j)} \in G$ We may assume $\pi g^{(j)}=$ $x_{j} \rightarrow x \in \Omega$ For $y$ large, $x_{j} \in$ Core $(x)$ and the $P_{0}^{(J)} \in \mathscr{P}\left(x_{j}\right) \subset \mathscr{P}(x)$ have a constant subsequence A standard diagonal argument gives a subsequence $j_{n} \rightarrow \infty$ so that

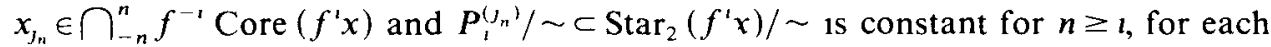
$\imath \geq 0$ Then $g^{\left(J_{n}\right)}$ converges in $G$

Thus we see that $G$ is compact and $h$ is an expansive homeomorphism We next show $h$ has CC Say $\left(g, g^{\prime}\right) \in V(z)$ and $x=\pi g$ is close to $x^{\prime}=\pi g^{\prime}$ With $y_{m n} \rightarrow x$ and $y_{m n}^{\prime} \rightarrow x^{\prime}$ as in the definition of $s$-germ we see by $(\beta)$ that $\left[y_{m n}, y_{m n}^{\prime}\right]=y_{m n}^{\prime \prime}$ is defined for $m \ll 0, n \gg 0$ Thus $x^{\prime \prime}=\lim y_{m n}^{\prime \prime}=\left[x, x^{\prime}\right]$ exists Let $g^{\prime \prime} \in G_{x^{\prime}}$ be $g^{\prime \prime}=\left(P_{\imath}^{\prime \prime}\right)$ where $P_{1}^{\prime \prime}$ is the value of $P\left(f^{\prime} y_{m n}^{\prime \prime}, f^{\prime} x^{\prime \prime}\right)$ for $m \ll 0, n \gg 0$ A little thought shows that $P_{1}^{\prime \prime}$ is well defined, that $g^{\prime \prime} \in G_{\mathrm{r}}$ and that $g^{\prime \prime}=\left[g, g^{\prime}\right]$ This gives a neighbourhood of $\Delta_{G}$ on which $[$,$] is defined so h$ has CC This finishes the proof of Lemma 3 , so Theorem 1 is proven

While the details of Lemma 3 are foul the 1dea is quite intuitive Consider, say, the case $\Omega$ a surface, $f$ pseudo-Anosov Then we must break up the prongs since canonical coordınates break down there But one cannot just alter the prongs one must split open their stable sets, as shown in Figure 1 This can be done by distınguishing the different sides of these stable sets, which are just $s$-germs The prong singularity $p$ has 3 nontrivial $s$-germs, other points $x \in W^{s}(p)$ have only 2 Note that the shaded region $X \subset G$ is the closure of the singleton fibers of $\pi X$ can be identified with the DA repeller obtained by converting prongs into sinks The canonical coordinates on $G$ and the $s$-resolving property of $\pi$ are plainly visible 


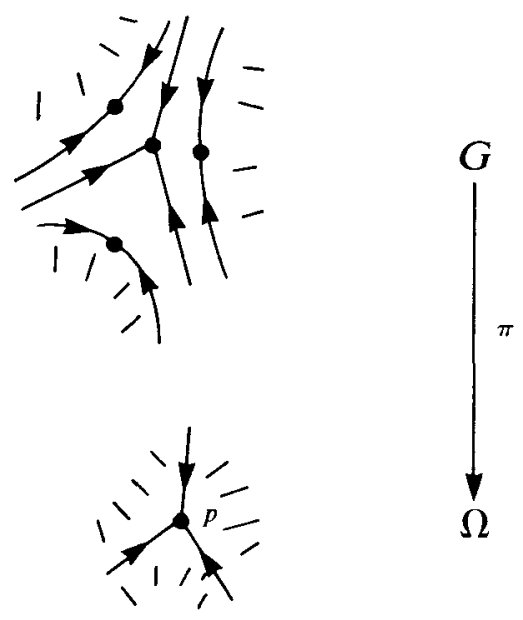

FIGURE 1

Remark 1 The $s$-germ extension $\pi \quad G \rightarrow \Omega$ constructed above is canonical First suppose $\delta$ is changed to $\delta^{\prime}<\delta$ Then the set $G$ is unchanged and the new uniformity is defined by a $V^{\prime}$ that clearly contains $V$ As a contınuous bijection of compact Hausdorff spaces is a homeomorphism we see that the topology of $G$ is independent of $\delta$ Second suppose one changes the cover $\left\{R_{s}\right\}$ it is enough to consider the case of adding a new rectangle Then the cores become smaller and the stars become larger so there is a restriction map $G^{\prime} \rightarrow G$ from new $s$-germs to old ones (here we fix $\delta$ very small) One easily can check that this map is bijective and continuous so $G$ is unchanged Likewise one can alter the choice of $d$ without changing $G$

For sofic systems ( $\mathrm{e} \operatorname{dim} \Omega=0$ ) this sort of extension is known but by explicit symbolic constructions [Kr] We believe the $s$-germs represent the latent geometry in the notion of future-resolving extensions of sofic systems

Now we will prove Theorem 2, 1 e MP $\Leftrightarrow$ FP If $\mathscr{M}$ is a Markov partition then to each sequence $R_{n} \in \mathcal{M}$ for which $f\left(\right.$ int $\left.R_{n}\right)$ meets int $R_{n+1}$ for all $n \in \mathbb{Z}$ there is a unique point $\pi\left(R_{n}\right) \in \bigcap_{n \in \mathbb{Z}} f^{-n} R_{n}$ These sequences form an SFT $\Sigma$ with symbol set $\mathcal{M}$ and $\pi$ represents $f$ as a factor This is proven just like the basic set case, e $g$ [B1, pp 84-86] In this case the equivalence relation $E$ on $\Sigma$ defined by $\pi$ is

$$
\left(R_{n}\right) E\left(R_{n}^{\prime}\right) \Leftrightarrow R_{n} \text { meets } R_{n}^{\prime}, \text { all } n \in \mathbb{Z}
$$

as noted by Bowen [B2, p 13] Clearly $E$ is an SFT Thus MP $\Rightarrow$ FP

Conversely, suppose we are given an expansive $f \Omega \rightarrow \Omega$ with expansive constant $c=2 \varepsilon$, an SFT $\sigma \quad \Sigma \rightarrow \Sigma$, and a semiconjugacy $\pi \quad \Sigma \rightarrow \Omega$ We will follow [B1, pp 7883 , to construct a Markov partition

By symbol splittıng ( $1 \mathrm{e}$ passıng to new symbols that are consecutive $N$-strings of old symbols, $N$ large) we may suppose that $\Sigma$ is defined by relations on consecutive pairs of symbols, 1 e by a graph $A \subset \mathscr{S} \times \mathscr{S}$ of allowed transitions

$$
\left(s_{n}\right) \in \Sigma \Leftrightarrow s_{n} A s_{n+1}, \quad n \in \mathbb{Z}
$$


We may also assume that for each $s \in \mathscr{P}$ the cylinder $C_{s}=\left\{\left(s_{n}\right) \in \Sigma \mid s_{0}=s\right\}$ has an image $T_{s}=\pi\left(C_{s}\right)$ in $\Omega$ of diameter $<\varepsilon / 2$ Then $T_{s}$ is a closed rectangle, since $\left(s_{n}\right)$, $\left(s_{n}^{\prime}\right) \in C_{s}$ imply

$$
\begin{gathered}
{\left[\pi\left(s_{n}\right), \pi\left(s_{n}^{\prime}\right)\right]=\pi\left(s_{n}^{*}\right),} \\
s_{n}^{*}= \begin{cases}s_{n}, & n \geq 0, \\
s_{n}^{\prime}, & n \leq 0\end{cases}
\end{gathered}
$$

The $T_{\mathrm{s}}$ clearly form a cover $\mathscr{T}$ of $\Omega$ Also if $x \in T_{s}$ and $s A t$ then the Markov property (M)

$$
f W_{\varepsilon}^{s}\left(x, T_{s}\right) \subset W_{\varepsilon}^{s}\left(f x, T_{t}\right), \quad f^{-1} W_{\varepsilon}^{u}\left(f x, T_{t}\right) \subset W_{\varepsilon}^{u}\left(x, T_{s}\right)
$$

holds We must, however, modify $\mathscr{T}$ to produce a Markov partition since we do not know whether the $T_{s}$ are proper or have disjoint interiors

Consider any closed rectangle $T$ We must analyze how $T$ decomposes relative to the cover $\mathscr{T}$ For $k \in \mathscr{S}$ let the unstable $k$-boundary of $T$ be

$$
\partial_{k}^{u} T=\left\{x \in T \mid x=\lim x_{i}, x_{i} \in W_{\varepsilon}^{s}(x) \cap T_{k}-T\right\}
$$

and define $\partial_{k}^{s} T$ accordingly (with $x_{1} \in W_{\varepsilon}^{u}(x)$ ) Then

$$
\partial T=\bigcup_{k}\left(\partial_{k}^{u} T \cup \partial_{k}^{s} T\right)
$$

For if $x \in \partial T$, say $x=\lim y_{1}, y_{1} \notin T$, then by passing to a subsequence we can assume all $y_{1} \in T_{k}$, some $k$ Then $x \in T_{k}$ and we can form $y_{t}^{\prime}=\left[x, y_{t}\right] \in T_{k}$ and $y_{t}^{\prime \prime}=\left[y_{1}, x\right] \in T_{k}$ Since $\left[y_{1}^{\prime \prime}, y_{1}^{\prime}\right]=y_{1} \notin T$ either $y_{1}^{\prime} \notin T$ or $y_{1}^{\prime \prime} \notin T$ Passing to a subsequence, assume say that $y_{1}^{\prime} \notin T$ for all $\imath$ Then $y_{1}^{\prime} \in W_{\varepsilon}^{s}(x) \cap T_{k}-T, y_{1}^{\prime} \rightarrow x$ so $x \in \partial_{k}^{u} T$ If $y_{1}^{\prime \prime} \notin T$ for all $l$ then $x \in \partial_{k}^{s} T$

From $T$ we form the quotient space $H$ in which $x, y \in T$ are identified if $x \in W_{\varepsilon}^{s}(y)$ and the corresponding space $V$ of $\varepsilon$-unstable sets The natural map $T \rightarrow H \times V$ is a homeomorphism and we will identify $T$ with $H \times V$ For $k \in \mathscr{G}$ define closed subspaces $H_{k}^{*} \subset H_{k} \subset H$ to be the projections of $\partial_{k}^{s} T \subset T \cap T_{k} \subset T$, and define $V_{k}^{*} \subset V_{k} \subset V$ accordingly Then one sees $\partial_{k}^{s} T=H_{k}^{*} \times V_{k}$ and $\partial_{k}^{u} T=H_{k} \times V_{k}^{*}$ are rectangles, as follows Say $x=\lim x_{i}, x_{i} \in W_{\varepsilon}^{u}(x) \cap T_{k}-T$, and $y \in T_{k} \cap T$ Ther. $\left[x_{i}, y\right] \notin T$, since otherwise $x_{1}=\left[\left[x_{1}, y\right], x\right] \in T$ Thus $[x, y]=\lim \left[x_{i}, y\right],\left[x_{i}, y\right] \epsilon$ $W_{\varepsilon}^{u}([x, y]) \cap T_{k}-T$, so $[x, y] \in \partial_{k}^{s} T$

Define rectangles $T_{k}^{n} \subset T$ by

$$
\begin{aligned}
& T_{k}^{i}=\operatorname{int}_{H}\left(H_{k}-H_{k}^{*}\right) \times \operatorname{int}_{V}\left(V_{k}-V_{k}^{*}\right) \\
& T_{k}^{2}=\operatorname{lnt}_{H}\left(H-H_{k}\right) \times \operatorname{nnt}_{V}\left(V_{k}\right) \\
& T_{k}^{3}=\operatorname{lnt}_{H}\left(H_{k}\right) \times \operatorname{int}_{V}\left(V-V_{k}\right) \\
& T_{k}^{4}=\operatorname{int}_{H}\left(H-H_{k}\right) \times \operatorname{int}_{V}\left(V-V_{k}\right)
\end{aligned}
$$

where int $_{H}$, int ${ }_{V}$ denote interior relative to $H, V$ respectively Then each $T_{k}^{n}$ is open as a subset of $T$ For $\nu \mathscr{S} \rightarrow\{1,2,3,4\}$ the rectangle $T^{\nu}=\bigcap_{k} T_{k}^{\nu(k)}$ is open in $T$ and disjoint from $\partial T=\bigcup_{k}\left(H_{k}^{*} \times V_{k}\right) \cup\left(H_{k} \times V_{k}^{*}\right)$, hence $T$ is open in $\Omega$ Also the error set

$$
\mathscr{E}(T)=\bigcup_{k \in \mathscr{S}}\left(T-\bigcup_{n=1}^{4} T_{k}^{n}\right)=T-\bigcup_{\nu} T^{\nu}
$$

1s closed, nowhere dense in $\Omega$ and contains $\partial T$ See Figure 2 

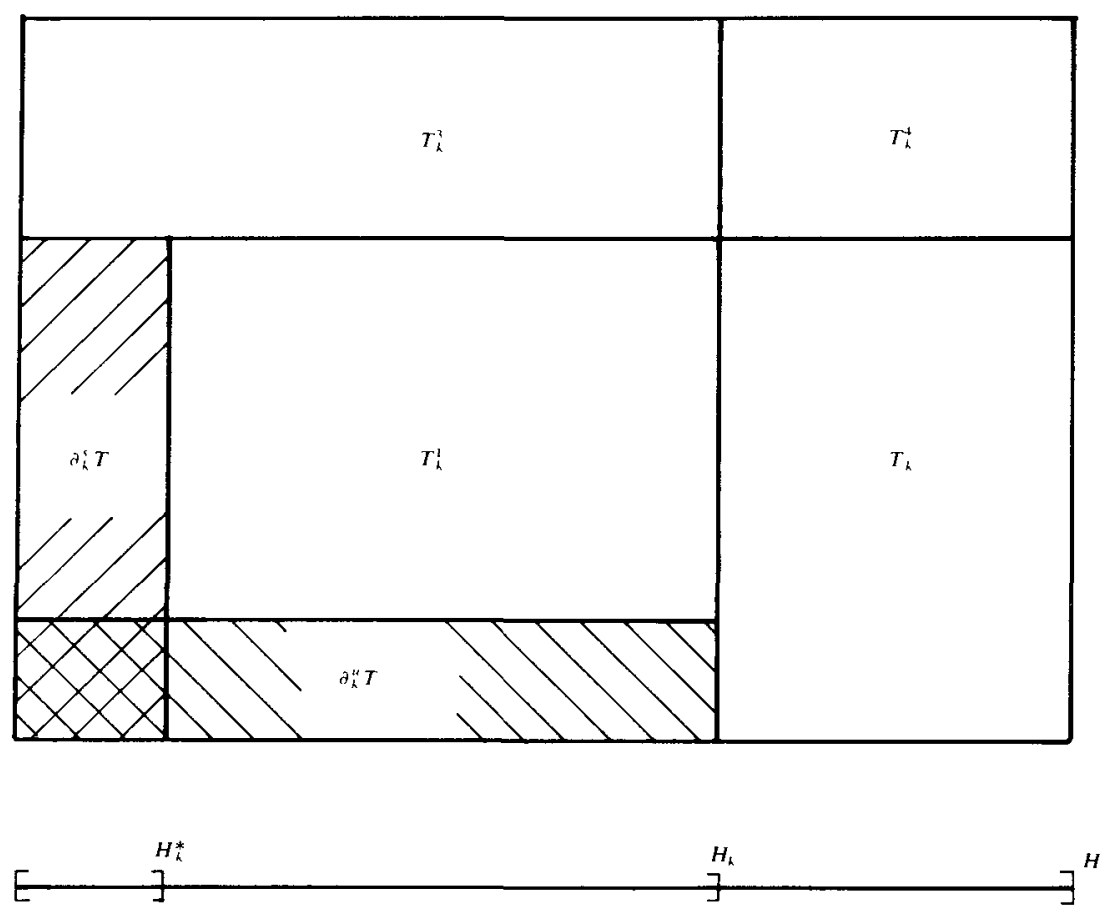

FIGURE 2

Now let $\mathscr{D}=\Omega-\bigcup_{J \in \mathscr{S}} \mathscr{E}\left(T_{J}\right)$ Then $\mathscr{D}$ is open and dense For $x \in \Omega$ we let $\mathscr{P}(x) \subset \mathscr{S}$ consist of those $J$ for which $x \in T$, Then

$$
\begin{aligned}
x \in \mathscr{D} & \Leftrightarrow \forall j \in \mathscr{S}(x), x \notin \mathscr{E}\left(T_{j}\right) \\
& \Leftrightarrow \forall J \in \mathscr{S}(x), k \in \mathscr{P}, \exists n=n(x, j, k) \text { such that } x \in\left(T_{j}\right)_{k}^{n}
\end{aligned}
$$

and for such $x$ the rectangle $R(x)$ defined by

$$
R(x)=\bigcap_{J \in \mathscr{S}(x)} \bigcap_{k \in \mathscr{S}}\left(T_{j}\right)_{k}^{\left.n\left(x_{J}\right) k\right)}
$$

1s open and contains $x$ We have (c $\mathrm{f}$ [B1, pp 81-82]

LemMA 4 For $x, y \in \mathscr{D}, R(x)$ and $R(y)$ are either disjoint or equal The set $\mathscr{R}=$ $\{\overline{R(x)} \mid x \in \mathscr{D}\}$ is a finite cover of $\Omega$ by proper rectangles with disjoint interiors

LEMMA 5 For $x, y \in \mathscr{D} \cap f^{-1} \mathscr{D}$ with $R(x)=R(y)$ and $y \in W_{\varepsilon}^{s}(x)$ one has $R(f x)=$ $R(f y)$

Proof of Lemma 4 If $z \in \mathscr{D} \cap R(x)$ then $\mathscr{S}(z)=\mathscr{P}(x)$ Also if $j \in \mathscr{P}(x)$ and $k \in \mathscr{S}$ then $z \in\left(T_{j}\right)_{k}^{n}$ for the same value of $n, 1$ e $n(x, j, k)=n(z, j, k)$, so $R(x)=R(z)$ Thus If $R(x) \cap R(y) \neq \varnothing$ there is a $z \in \mathscr{D} \cap R(x) \cap R(y)$ so that $R(x)=R(z)=R(y)$

Proof of Lemma 5 First we write $f x=\pi\left(\sigma\left(q_{n}\right)\right)$ where $q_{1}=\jmath, q_{0}=s$ Thus $f x \in T_{j}$, $x \in T_{s}$ As $y \in W_{\varepsilon}^{s}\left(x, T_{s}\right)$, (M) implies $f y \in W_{\varepsilon}^{s}\left(f x, T_{j}\right)$ So $f x \in T_{j} \Rightarrow f y \in T_{j}$, and, by symmetry, $\mathscr{P}(f x)=\mathscr{S}(f y)$ 
Next we show $n(f x, j, k)=n(f y, J, k)$ for $k \in \mathscr{S}$ As $W_{\varepsilon}^{s}\left(f y, T_{\jmath}\right)=W_{\varepsilon}^{s}\left(f x, T_{J}\right)$ we see these $n$ 's are of the same parity We must show that if $W_{\varepsilon}^{u}\left(f x, T_{j}\right)$ meets $T_{k}$ then the same holds for $f y$ by symmetry, this will prove the lemma

Say $f z \in T_{k} \cap W_{\varepsilon}^{u}\left(f x, T_{j}\right)$ By $(\mathrm{M}), z \in W_{\varepsilon}^{u}\left(x, T_{s}\right)$ As $f z \in T_{k}$ we have $z=\pi\left(q_{n}^{\prime}\right)$ with $q_{1}^{\prime}=k$ Let $t=q_{0}^{\prime} \in \mathscr{P}$ Again (M) gives

$$
f W_{\varepsilon}^{s}\left(z, T_{t}\right) \subset W_{\varepsilon}^{s}\left(f z, T_{k}\right)
$$

Since $T_{s} \in \mathscr{P}(x)=\mathscr{P}(y)$ and $T_{t}$ meets $W_{\varepsilon}^{u}\left(x, T_{s}\right)$ at $z, T_{t}$ also meets $W_{\varepsilon}^{u}\left(y, T_{s}\right)$ at some point $z^{\prime}$ Let $z^{\prime \prime}=[z, y]$ Then $z^{\prime \prime}=\left[z, z^{\prime}\right] \in W_{\varepsilon}^{s}\left(z, T_{t}\right) \cap W_{\varepsilon}^{u}\left(y, T_{s}\right)$ In $T_{j}$ then $f z^{\prime \prime}=[f z, f y] \in W_{\varepsilon}^{s}\left(f z, T_{k}\right) \cap W_{\varepsilon}^{u}\left(f y, T_{j}\right)$, showing $W_{\varepsilon}^{u}\left(f y, T_{j}\right)$ meets $T_{k}$, as desired

By Lemma 4 we must only show $\mathscr{R}$ has the Markov property For $R_{1}, R_{2} \in \mathscr{R}$ (i $\mathrm{e}$ $\left.R_{1}=\overline{R\left(p_{1}\right)}, p_{1} \in \mathscr{D}\right), f^{-1}$ (1nt $R_{2}$ ) meets int $R_{1} \Leftrightarrow f^{-1} R\left(p_{2}\right)$ meets $R\left(p_{1}\right)$ By continuity and symmetry (1 e arguing with $f^{-1}$ instead of $f$ ) it suffices to show for $x \in$ $\left(R\left(p_{1}\right) \cap f^{-1} R\left(p_{2}\right)\right)=\mathscr{O}$

$$
f W_{\varepsilon}^{s}\left(x, R_{1}\right) \subset R_{2}
$$

Say $z \in W_{\varepsilon}^{s}\left(x, R_{1}\right)$ Write $x=\lim w_{1}, w_{1} \in \mathscr{O} \cap \mathscr{D} \cap f^{-1} \mathscr{D}(\mathcal{O}$ is open and $\mathscr{D}$ is dense) Then $\left[w_{1}, z\right] \rightarrow z$ Choose $y_{i}$ very near $\left[w_{1}, z\right]$ so $y_{1} \in R\left(p_{1}\right) \cap \mathscr{D} \cap f^{-1} \mathscr{D}$ and so that $x_{1}=\left[y_{1}, w_{1}\right]$ is still in $\mathscr{O} \cap \mathscr{D} \cap f^{-1} \mathscr{D}$ Then $y_{1} \rightarrow z, x_{1} \rightarrow x$ and Lemma 6 above, applied to $x_{1}$ and $y_{1}$, shows $f y_{1} \in R\left(p_{2}\right)$ I hus $f z=11 \mathrm{~m} f y_{t} \in R_{2}$, as was to be shown

While this proof is substantially the same as the Bowen argument we note some differences The major problem is that if $T$ is a rectangle int $(T)$ may not be a rectangle' Indeed although each $\partial_{k}^{s} T$ is a rectangle there may be $x \in W_{\varepsilon}^{s}(y, T)$,

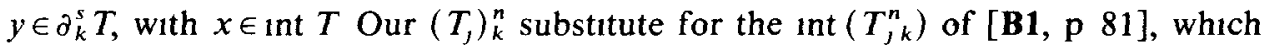
may not be rectangles in our setup Also the set $Z^{*}$ of [B1, p 80], may not be dense here so we have to use $\mathscr{D}$ instead

\section{Cohomology and periodic points}

We will introduce a refined symbolic dynamics to study the topology of a factor of a subshift, especially its Cech cohomology This will enable us to analyze Manning's counting argument in terms of coincidence numbers A useful reference for this section is the classic book of Ellenberg and Steenrod [ES]

Let $\Sigma$ be a subshift on symbol set $\mathscr{S}$ and $\Omega$ a factor of $\Sigma$ For $a \leq b, a, b \in \mathbb{Z}$, define an $(a, b)$ cylinder set $v \subset \Sigma$ to be a nonempty subset of $\Sigma$ obtained by specifying the sequence $\left(s_{n}\right) \in \Sigma$ for $n=a, \quad, b$ These form a partition $\mathscr{S}_{a b}$ of $\Sigma$ whose images form a finite cover $\mathscr{T}_{a b}$ of $\Omega$ by compact sets $\pi(v)$ indexed by $v \in \mathscr{S}_{a b}$ Let $N_{a b}$ be the nerve of $\mathscr{T}_{a b}$, so $N_{a b}$ is the finite simplicial complex with one J-simplex for each $(J+1)$-element subset of $\mathscr{S}_{a b}$ whose images under $\pi$ have nonempty intersection Let $F_{a b}$ be the filled-in complex with one $j$-simplex for every $(j+1)$-element subset of $\mathscr{S}_{a b}$ whose images under $\pi$ intersect pairwise The 1-skeleton $\Gamma_{a b}$ of $N_{a b}$ is also the 1-skeleton of $F_{a b}$ and $\Gamma_{a b}$ determines $F_{a b}$ since a subset of $\mathscr{S}_{a b}$ spans a simplex in $F_{a b}$ if and only if its two element subsets span an edge in $\Gamma_{a b}$ We have

$$
\mathscr{S}_{a b} \subset \Gamma_{a b} \subset N_{a b} \subset F_{a b}
$$

If $\alpha \leq a \leq b \leq \beta$ then $\mathscr{S}_{\alpha \beta}$ refines $\mathscr{S}_{a b}, \mathscr{T}_{\alpha \beta}$ refines $\mathscr{T}_{a b}$ and one has a simplicial map $\phi_{a b}^{\alpha \beta} N_{\alpha \beta} \rightarrow N_{a b}$ These maps form an inverse system indexed by integer pairs 
$a \leq b$ and we form the inverse limit space $N=\lim _{\leftarrow} N_{a b}$ in which a point $p=\left(p_{a b}\right)$ consists of a point $p_{a b} \in N_{a b}$ for all $a \leq b$ such that $\phi_{a b}^{\alpha \beta} p_{\alpha \beta}=p_{a b}$ As the maps in the inverse system preserve skeleta $N$ is filtered by the inverse limits $N^{\prime}$ of the l-skeleta $N_{a b}^{1}$ Since $N_{a b}^{0}=\mathscr{F}_{a b}$ we see that $N^{0}=\Sigma$ is the shift we began with We can likewise form the inverse system of simplicial maps $\psi_{a b}^{\alpha \beta} F_{\alpha \beta} \rightarrow F_{a b}$ and take its inverse limit to obtain a space $F$, filtered by $F^{i}=\lim _{\leftarrow} F_{a b}^{l}$ We have so far

$$
\begin{array}{ll}
\Sigma \subset N^{1} \subset N^{2} \subset & \subset N \\
\|\|\|\quad\| & \cap \\
\Sigma \subset F^{1} \subset F^{2} \subset & \subset F
\end{array}
$$

We can obtain more by embedding $F$ in the space $M \Sigma$ of regular Borel measures on $\Sigma$ as follows To $q=\left(q_{a b}\right) \in F$ we associate the measure $\mu_{q} \in M \Sigma$ whose value on the cylinder set $v \in \mathscr{S}_{a b}$ is the barycentric coordinate of $q_{a b}$ at the vertex $v$

$$
\mu_{q}(v)=q_{a b}(v) \quad\left(a, b \in \mathbb{Z}, a \leq b, v \in \mathscr{Y}_{a b}\right)
$$

Since barycentric coordinates are a probability measure on the vertex set $\mathscr{Y}_{a b}$ and since $\psi_{a b}^{\alpha \beta} q_{\alpha \beta}=q_{a b}$, those assignments of measures to cylinder sets satisfy Kolmogorov's consistency conditions ([DGS, p 41]) and so determine a measure on $\mathscr{G}^{\mathbb{Z}}$ with support on $\Sigma$, which gives the desired $\mu_{q}$

Let Supp $\left(q_{a b}\right) \subset \mathscr{S}_{a b}$ be the support of the measure $q_{a b}$ Then the set

$$
\tau_{a b}=\bigcup_{i \in \operatorname{Supp}\left(q_{a b}\right)} \pi(v) \subset \Omega
$$

has small diameter if $b \gg 0 \gg a$ since then each $\pi(v)$ has small diameter and any two $\pi(v)$ in this union intersect If $\alpha \leq a \leq b \leq \beta$ then $\tau_{\alpha \beta} \subset \tau_{a b}$ Thus there is a unique point $\xi(q) \in \Omega$ that lies in all the $\tau_{a b}, a \leq b$ One sees easily that the pushed forward measure $(M \pi) \mu_{q}$ on $\Omega$ is the point mass at $\xi(q)$ If $M_{\pi} \subset M \Sigma$ denotes the closed set of measures on $\Sigma$ that are supported on level sets of $\pi$ then we see that $\mu_{q} \in M_{\pi}$ for all $q \in F$

Next given a measure $\mu \in M_{\pi}$ we let $p_{a b}$ be the probability measure on $\mathscr{S}_{a b}$ with $p_{a b}(v)=\mu(v)$ Then $\operatorname{Supp}\left(p_{a b}\right) \subset\{v \mid x \in \pi(v)\}$ so $p_{a b} \in N_{a b}$ Clearly the point $p_{\mu}=$ ( $p_{a b}$ ) belongs to $N$ and defines an embedding of $M_{\pi}$ in $N$ We have shown altogether that $F \simeq N \simeq M_{\pi}$ A little thought shows that the subspace $M_{\pi}^{\prime}$ of measures with support on at most $l+1$ points and contained in some level set of $\pi$ is closed and that our isomorphisms identify $F^{\prime}$ and $N^{i}$ with $M_{\pi}^{\prime}$ We have then (1dentifying $F$ with $N$ )

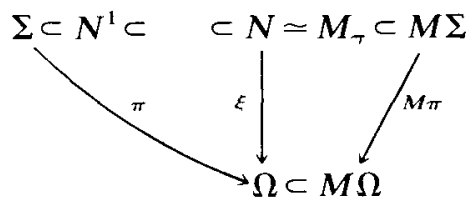

This $\xi$ is the refined symbolic dynamics mentıoned earlier A level set $\xi^{-1}(x)$ consists of all the formal averages of the symbolic names of a point $x, 1 \mathrm{e}$ the simplex with vertices $\pi^{-1}(x)$

The advantage of $\xi$ over $\pi$ is that it induces an isomorphism $\xi^{*}$ on Čech cohomology (with any coefficient group $G$ ) Namely the covers $\tau_{a b}$ have mesh that 
tends to zero as $a \rightarrow-\infty, b \rightarrow+\infty$ and so

$$
\check{H}^{*} \Omega=\lim _{\rightarrow} H^{*} N_{a b}=\check{H}^{*} N
$$

by the continuity property of Čech cohomology The advantage of $F$ over $N$ is that $F$ is a prior determined by the inverse system of graphs $\Gamma_{a b}$, so higher order intersections can be ignored when computıng $\check{H}^{*} \Omega=\lim _{\rightarrow} H^{*} F_{a b}$

Of course the cohomology $\check{H}^{*} \Omega$ carries more structure than that of a group (or algebra, if $G$ is a ring) namely it has an automorphism $f^{*}$ This can also be identified in these inverse limits as the cohomology automorphism induced by the shift homeomorphism

$$
\left(p_{a b}\right) \mapsto\left(p_{a+1 b+1}\right)
$$

of $N$ We can group the $N_{a b}$ with a fixed value of $b-a$ together and identify $H^{*} N_{a b}$ with $t^{a} H_{0, b-a}^{*}$ for some indeterminate $t$ to obtain

$$
\check{H}^{*} \Omega=\lim _{\rightarrow} H^{*} N_{0 p}\left[t, t^{-1}\right]
$$

where the maps in the limit are of the form $A_{p}+t B_{p}$, with $A_{p}, B_{p} H^{*} N_{0 p} \rightarrow H^{*} N_{0 p+1}$ induced by the simplicial maps

$$
N_{0 p+1} \rightarrow N_{0 p}, \quad N_{0 p+1} \rightarrow N_{1 p+1} \approx N_{0 p}
$$

respectively Here the action of $f^{*}$ on $\check{H}^{*} \Omega$ corresponds to multiplication by $t$ on the direct limit So, replacing $N_{a b}$ by $F_{a b}$ throughout, we see that the inverse system of graphs $\Gamma_{a b}$ determınes $\check{H}^{*} \Omega$ and the action of $f^{*}$

Also one has a cochain complex for computıng $\check{H}^{*} \Omega$ whose $t$ th group is

$$
\check{H}^{i}\left(M_{\pi}^{i}, M_{\pi}^{i-1}\right)=\lim _{\rightarrow} C^{l}\left(N_{a b}\right)=\lim _{\rightarrow} C^{t}\left(F_{a b}\right)
$$

and whose coboundary operators arise from the triples $\left(M_{\pi}^{i+1}, M_{\pi}^{i}, M_{\pi}^{i-1}\right)$ Thus the 'skeleta' $M_{\pi}^{\prime}$ play the same role as the skeleta of a finite complex for cohomology computations Note that these relative cohomology groups carry the $f^{*}$ action, unlike the finitely generated groups $C^{\prime}\left(N_{a b}\right)$ and $C^{\prime}\left(F_{a b}\right)$

As an application we study the space $C \Omega$ of connected components of $\Omega, 1$ e the largest zero-dimensional quotient of $f$ By Stone's theorem $C \Omega$ is the space of maximal ideals in the Boolean algebra of closed-open subsets of $\Omega$, which in turn is just the cohomology group $H^{0}\left(\Omega, \mathbb{Z}_{2}\right)$ Thus the above cohomology computation implicitly computes $C \Omega$ and one obtains easily the explicit formula

$$
C \Omega=\lim _{\longleftarrow} C\left(\Gamma_{a b}\right)
$$

Here the map $C f$ on components corresponds to the shift map $\left(p_{a b}\right) \mapsto\left(p_{a+1, b+1}\right)$ on the inverse limit For $m \geq 0$ we identify all the $C\left(\Gamma_{a b}\right)$ with $b-a=m$ with some finite set $S_{m}$, so $\left(p_{a b}\right)$ determines a point $\left(p_{n n+m}\right) \in S_{m}^{\mathbb{Z}}$ This defines a factor $\Sigma_{m}$ of $C \Omega$ that is a subshift on symbol set $S_{m}$ One clearly has

$$
C \Omega=\lim _{\leftarrow} \Sigma_{m}
$$

and so $C f$ is an inverse limit of subshifts Note that each map $\Sigma_{m+1} \rightarrow \Sigma_{m}$ is surjective

We now show that $C \Omega$ is expansive $\Leftrightarrow \Sigma_{m}$ is eventually constant More generally, given an inverse system of expansive systems $X_{m}$ with $X_{m+1} \rightarrow X_{m}$ surjective, the inverse limit $X$ is expansive $\Leftrightarrow X_{m+1} \rightarrow X_{m}$ is bijective for $m$ sufficiently large For consider the equivalence relation $E_{m} \subset X \times X$ defined by the surjective $X \rightarrow X_{m} E_{m}$ 
is isolated, $E_{m+1} \subset E_{m}$ and $\bigcap E_{m}=\Delta$ is the diagonal in $X \times X$ Thus $\Delta$ is isolated $\Leftrightarrow \Delta=$ $E_{m}$ for $m$ large, as desired

This criterion suggests that for most FP $\Omega$ the system $C \Omega$ is not expansive, yet no example of this behavior is in the literature In particular all the usual Axiom A basic sets have an SFT as their component map Indeed if $\Omega$ has canonical coordinates and $C \Omega$ is expansive then $C \Omega$ is an SFT

Counterexamples can be found by using a construction of Guckenheimer Begin with the Julia set $J$ for a hyperbolic rational map $f$ and form the inverse limit $\Omega$ of $\quad \rightarrow J \stackrel{f}{\rightarrow} J \stackrel{f}{\rightarrow} J$ Then $\Omega$ is a basic set [G2] Shishikura has shown us, however, that for $f$ a polynomial, $C \Omega$ is expansive $\Leftrightarrow$ either $J$ is connected or $J$ is zero dimensional In all other cases there is an infinite component $Y$ of $J$ which surrounds a critical point of $f$, and one can choose a one-point component $\{z\} \subset J$ arbitrarily near $f Y$ Then $z$ has distinct preimages $z^{\prime}, z^{\prime \prime}$ which lie very near $Y$ In $C J$ the points $z^{\prime}, z^{\prime \prime}$ are very close and become equal after one iterate, so $C J$ is not positively expansive Even upon passage to the inverse limit certain prehistories of $z^{\prime}, z^{\prime \prime}$ stay very close in $C \Omega$ and so violate expansiveness

Note that for $\operatorname{deg} f$ large the generic behavior seems to be for $C \Omega$ not to be expansive, as suggested by the above analysis The symbolic description of $C J$ for cubic polynomials has been carried out by Blanchard and Branner-Hubbard, where already this nonexpansive behaviour arises $[\mathrm{BI}],[\mathrm{BH}]$

Now we consider the FP case and show how to compute cohomology from transition and incidence data Suppose $\Omega=\Sigma_{A} / E$ where $A \subset \mathscr{S} \times \mathscr{S}$ is a transition relation and where the equivalence relation $E=E_{I}$ is

$$
\left(s_{n}\right) E\left(s_{n}^{\prime}\right) \Leftrightarrow s_{n} I s_{n}^{\prime}, \quad \text { all } n \in \mathbb{Z},
$$

where $I \subset \mathscr{S} \times \mathscr{S}$ is a generalized incidence relation We do not require that $I$ be the actual incidence relation defined by the cover of $\Omega$ by images of cylinder sets in $\mathscr{S}_{\infty}$ (c $\mathrm{f}$ the proof of Theorem 2 ), although $I$ must clearly include the latter relation There is a certain compatability necessary between $I$ and $A$ to make $E$ an equivalence relation, a situation analyzed in [F3]

A cylınder set $v \in \mathscr{S}_{a b}$ corresponds to a sequence of specified values $s_{n} \in \mathscr{P}$, $a \leq n \leq b$, with $s_{n} A s_{n+1}, a \leq n \leq b$ We denote this by $v\left(s_{a}, \quad, s_{b}\right)$ Define a symmetric, reflexive relation $I_{a b}$ on $\mathscr{S}_{a b}$ by

$$
v\left(s_{a}, \quad, s_{b}\right) I_{a b} v\left(s_{a}^{\prime}, \quad, s_{b}^{\prime}\right) \Leftrightarrow s_{n} I s_{n}^{\prime}, \quad a \leq n \leq b
$$

Then $I_{a b}$ determines a 1-complex $\tilde{\Gamma}_{a b}$ with vertex set $\mathscr{S}_{a b}$ and an edge joining $v, v^{\prime} \in \mathscr{T}_{a b}$ if and only if $v \neq v^{\prime}$ and $v I_{a b} v^{\prime}$ Clearly $\Gamma_{a b} \subset \tilde{\Gamma}_{a b}$ We fill in $\tilde{\Gamma}_{a b}$ to obtain a finite complex $K_{a b}$ with an $l$-simplex for every set of $t+1$ vertices $v$ any two of which are $I$ related Clearly $F_{a b} \subset K_{a b}$ and $\tilde{\Gamma}_{a b}$ is the 1-skeleton of $K_{a b} \tilde{\Gamma}_{a b}$ and $K_{a b}$ are inverse systems of simplicial complexes and one sees easily that

$$
\lim \Gamma_{a b} \subset \lim _{\leftarrow} \tilde{\Gamma}_{a b} \subset M_{\pi}^{1}
$$

For the relation $s_{n} I s_{n}^{\prime}, n \in \mathbb{Z}$, implies $\pi\left(s_{n}\right)=\pi\left(s_{n}^{\prime}\right)$ and so the measure determined by a point $\left(r_{a b}\right) \in \lim _{\leftarrow} \tilde{\Gamma}_{a b}$ is supported on at most 2 points in the same fiber of $\pi$ 
Thus $\lim _{\leftarrow} \tilde{\Gamma}_{a b}=M_{\pi}^{1}$ Similarly

$$
\lim _{\longleftarrow} K_{a b}^{i}=M_{\pi}^{i}, \quad \lim _{\leftarrow} K_{a b}=M_{\pi}
$$

and consequently

$$
\check{H}^{*} \Omega=\lim _{\rightarrow} H^{*} K_{a b}=\lim _{\rightarrow} H^{*} K_{0 p}\left[t, t^{-1}\right]
$$

This gives an expression for $\check{H}^{*} \Omega$ in terms of the incidence and transition data $I$, $A$ alone, in which $f^{*}$ corresponds as before to multiplication by $t$

Now consider a simplicial map $L \quad X \rightarrow Y$ of two finite simplicial complexes For each $t \geq 0$ one has induced maps $L_{1}^{*} C^{\prime}(Y) \rightarrow C^{\prime}(X), L_{1 *} C_{1}(X) \rightarrow C_{1}(Y)$ and natural identifications $C^{t}(Y)=C_{i}(Y), \quad C^{t}(X)=C_{i}(X)$ where $C^{\imath}$ denotes $l^{-}$ dimensional cochains, $C_{t}$ denotes $t$-dimensional chains and we use $\mathbb{Z}$ coefficients Then if $R \quad X \rightarrow Y$ is another simplicial map we can define $(L, R)_{1}=\operatorname{Tr} L_{t}^{*} R_{i *}=$ $\operatorname{Tr} L_{i *} R_{i}^{*}$ to be the 1 -dimensional inner product of $L$ and $R$ The alternating sum $\#(L, R)=\Sigma(-1)^{\prime}(L, R)$, is the coincidence number of the simplicial correspondence $Y \stackrel{L}{\leftarrow} X \stackrel{R}{\rightarrow} Y$ If the maps in question are understood we write $\#(T \leftarrow X \rightarrow Y)$ for this coincidence number

In our settıng the most important case is $Y=K_{a a}=K_{b b}, X=K_{a b}$ for $a \leq b, L_{1 \mathrm{~s}}$ the natural map $K_{a b} \rightarrow K_{a a}$, and $R$ is the natural map $K_{a b} \rightarrow K_{b b}$ If $m=b-a$ we write $\#_{m}$ for $\#\left(K_{a a} \leftarrow K_{b b} \rightarrow K_{b b}\right)$

There is in fact a commutative diagram of simplicial maps of finite complexes where the left-bound arrows represent the maps $L$ and right-bound arrows represent

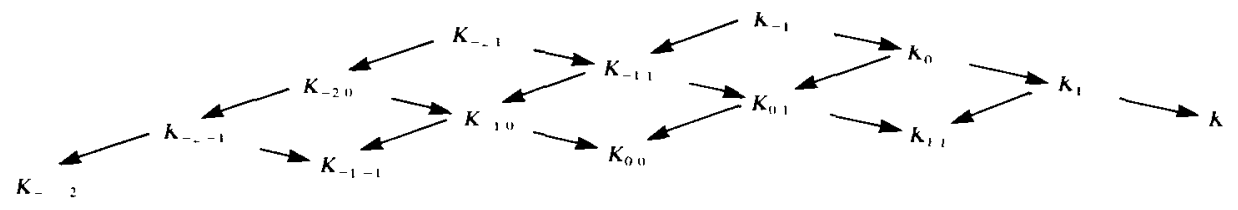

FIGURE 3

the maps $R$ (Figure 3 ) The numbers $\#_{m}$ measure coincidences between complexes on the bottom row with spacing $m$, as we shall see

Consider the diagram

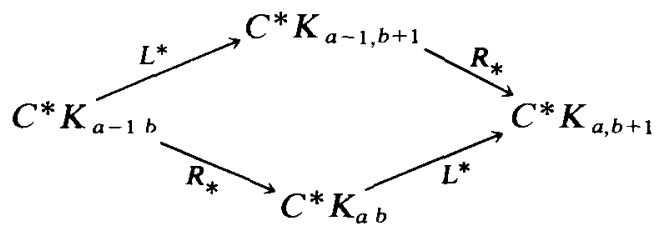

This diagram commutes thanks to the construction of $K$ For instance a 1 -simplex in $K_{a-1, b}$ is a figure

$$
\begin{array}{cc}
s_{a-1} A s_{a} A & A s_{b} \\
I \quad I & I \\
s_{a-1}^{\prime} A s_{a}^{\prime} A & A s_{b}^{\prime}
\end{array}
$$

where the horizontal rows are $A$ related and the vertical columns are $I$ related, as 
shown This maps (either through $R_{*} L^{*}$ or $L^{*} R_{*}$ ) to the sum of all 1-simplices

$$
\begin{aligned}
& s_{a} A \quad A s_{b} A s_{b+1} \\
& I \quad I \quad I \\
& s_{a} A \quad A s_{b}^{\prime} A s_{b+1}^{\prime}
\end{aligned}
$$

for $s_{b+1}, s_{b+1}^{\prime} \in \mathscr{S}$ satisfying the indicated $A, I$ relations

This commutativity allows one to replace the inverted $V$ composition $L^{*} L^{*} \quad L^{*} R_{*} R_{*} \quad R_{*}(m R$ 's, $m L$ 's $)$ in the definition of $\#_{m}$ by the zigzag $L^{*} R_{*} L^{*} R_{*} \quad L^{*} R_{*}$ Under our identifications of all the $\tilde{F}_{a a}$ to one complex we can view $L^{*} R_{*}$ as a chain map of the complex $K_{00}$ and find

$$
\#_{m}=\sum(-1)^{t} \operatorname{Tr}\left(L_{i}^{*} R_{t *}\right)^{m}
$$

The right hand side of this expression resembles a Lefschetz number of the $m$ th iterate of a map it 1s, however, the coincidence number of the $m$ th iterate of the self-correspondence

$$
K_{00} \leftarrow K_{01} \rightarrow K_{11}=K_{00}
$$

This alternating sum is exactly that found by Bowen [B2] in his interpretation of the Manning formula for the number of fixed points of $f^{m}$ We now have a chain level interpretation of this sum as a coincidence number and must explain why it in fact counts fixed points To do so, we must assume that $\pi \Sigma_{A} \rightarrow \Omega$ has finte fibers

Consider again a simplicial self-correspondence $X \stackrel{L}{\leftarrow} Y \stackrel{R}{\rightarrow} X$ Define the coincidence set $\{y \in Y \mid L y=R y\}=\mathscr{C}(L, R)=\mathscr{C}(X \leftarrow Y \rightarrow X) \quad \mathscr{C}(L, R)$ meets every simplex of $Y$ in a convex subspace and $Y$ could be subdivided so as to make $\mathscr{C}(L, R)$ a subcomplex Instead we consider the smallest subcomplex $\hat{\mathscr{C}}(L, R)$ of $Y$ that contains $\mathscr{C}(L, R)$

LEMMA 6 If $\hat{\mathscr{C}}(L, R)$ is the union of disjoint simplices $\Delta_{l}, t=1, \quad, k$, and $L, R$ are 1-1 on each $\Delta_{i}$ then \# $(L, R)=k$ is the number of components of $\mathscr{C}(L, R)$

Proof One immediately reduces to the case $Y=\bigcup \Delta_{i}$, since other simplices contribute neither to $\mathscr{C}(L, R)$ nor to $\#(L, R)$ Then one must have $L \Delta_{l}=R \Delta_{1}$ since $L \Delta_{1} \cap R \Delta_{1}$ contains $L\left(\Delta_{1} \cap \mathscr{C}(L, R)\right)$ and no subsimplex of $\Delta_{1}$ contains $\Delta_{1} \cap \mathscr{C}(L, R)$ By the Lefschetz fixed point formula applied to the simplicial 1somorphism $R L^{-1}$ of the simplex $L \Delta_{t}, \Delta_{i}$ contributes 1 to $\#(L, R)$, as desired

Note that we used exactly the trivial case of the Lefschetz formula that Bowen predicted would help topologize the Manning formula [B2] For a related application of the Lefschetz formula for a simplex to Axiom A flows see [F1] Note that simplicial maps $L \neq R$ from a 3-simplex $Y$ to a 1 -simplex $X$ that are two to one on vertices have $\#(L, R)=2$, so one must assume $L, R 1-1$ on $\Delta$, in Lemma 6

We now show that Lemma 6 applies to the correspondence $K_{00} \stackrel{L}{\leftarrow} K_{0 m} \stackrel{R}{\rightarrow} K_{m m}=$ $K_{00}$ and that each component of $\mathscr{C}(L, R)$ corresponds to a fixed point of $f^{m}$ Take a coincidence point $y \in K_{0 m}$ The support of $y$ consists of certain vertices $v_{1}=$ $\left(v_{0}^{(1)}, \quad, v_{m}^{(i)}\right) \in \mathscr{P}_{0 m}$, and the following coincidence condition holds

$$
\sum y\left(v_{t}\right) v_{0}^{(1)}=\sum y\left(v_{t}\right) v_{m}^{(t)}
$$

Take some $v_{1}$ for which $y\left(v_{t}\right)$ is smallest By the above equation $v_{m}^{(t)}$ is $v_{0}^{(j)}$ for some 
$J, v_{m}^{(j)}$ is $v_{0}^{(k)}$ for some $k$, etc By forming a nonrepeating cycle $\imath, \jmath, k, \quad, \imath$ and subtracting $y\left(v_{t}\right)\left(v_{0}^{(i)}+v_{0}^{(j)}+v_{0}^{(k)}+\quad\right)$ from both sides one obtains a similar equation with fewer terms In this way one finds that $y$ is a convex combination of certain barycenters $b$ with Supp $b \subset \operatorname{Supp} y$, and where each $b$ has the form

$$
b=\frac{1}{n} \sum_{l} v_{l} \quad l \in \operatorname{Supp} b, \quad n=|\operatorname{Supp} b|
$$

and where all the $v_{0}^{(l)}$ are distinct and equal (as a set) to the $v_{m}^{(l)}$ Then $b$ determines a cycle of transitions of length $m n$ by concatenating the sequences $v_{0}^{(l)}, \quad, v_{m}^{(l)}=$ $v_{0}^{(l)}, \quad, v_{m}^{(l)}, \quad$ and hence a periodic point $z$ in $\Sigma_{A}$ fixed by $\sigma^{m n}$ The points $z, \sigma^{m} z, \quad, \sigma^{m(n-1)} z$ are a periodic orbit of $\sigma^{m}$ that projects to a periodic orbit of $f^{m}$ but by expansiveness (in the form $\left(s_{l}\right) I\left(s_{l}^{\prime}\right)$ all $l \Rightarrow \pi\left(s_{l}\right)=\pi\left(s_{l}^{\prime}\right)$ ) this orbit is in fact a single point $x_{b} \in \mathrm{Fix}\left(f^{m}\right)$ For the same reason the fixed points $x_{b}$ arising from the various barycenters occurring in $y$ must all be equal, so $x_{b}=x_{y}$ The coincidence points $y$ with a given value of $x_{3}=x$ must lie in the simplex $\Delta_{x} \subset N_{0 m}$ spanned by the various cylinders that meet $\pi^{-1} x$ By expansiveness $\Delta_{x}$ and $\Delta_{x^{\prime}}$ are disjoint for $x, x^{\prime} \in \mathrm{F}_{1 \mathrm{X}}\left(f^{m}\right), x^{\prime} \neq x$ Conversely each $x \in \mathrm{F}_{1 \mathrm{X}}\left(f^{m}\right)$ is covered by a periodic point of $\sigma^{m}$ which determines a barycenter $b \in \Delta_{x} \cap \mathscr{C}(L, R)$ So fixed points correspond to components of $\mathscr{C}(L, R)$ and we have shown all but the assertion that $L, R$ are $1-1$ on each simplex in $\hat{\mathscr{C}}(L, R)$

Only here must we use our assumption that $\pi$ is finite to one If say $L$ is not 1-1 on the set $\Delta_{x} \cap \hat{\mathscr{C}}(L, R)$ then $\Delta_{x}$ contains two barycenters as above

$$
b=\frac{1}{n} \sum v_{l} \quad b^{\prime}=\frac{1}{n^{\prime}} \sum v_{l}
$$

with $v_{0}^{(l)}=v_{0}^{(l)}$ for some $l, l^{\prime}$ But then one can splice the periodic sequences in $\Sigma$ corresponding to $b, b^{\prime}$ at any common occurrence of $v_{0}^{(l)}$ to obtain infinitely many distinct sequences in $\pi^{-1} x$, a contradiction Thus we have (with $A_{t}=L_{t}^{*} R_{t *}$ )

THEOREM 3 For $\Omega=\Sigma_{A} / E_{I}$ with the equivalence classes of $E_{I}$ finite

$$
\left|\mathrm{F}_{1 \mathrm{X}} f^{m}\right|=\left|C_{\mathscr{C}}\left(K_{00} \leftarrow K_{0 m} \rightarrow K_{m m}\right)\right|=\#_{m}=\sum(-1)^{i} \operatorname{Tr}\left(A_{1}\right)^{m}
$$

Moreover $\zeta_{f}(t)=\Pi_{1} \operatorname{det}\left(I-t A_{\imath}\right)^{(-1)^{t+1}}$ is rational in $t$

Only the last equation needs to be shown but it follows as usual from the standard algebraic result

$$
\exp \sum \operatorname{Tr}\left(B^{m}\right) \frac{t^{m}}{m}=\operatorname{det}(I-t B)^{-1},
$$

together with the Manning-Bowen formula

$$
\left|F_{1 x} f^{m}\right|=\sum(-1)^{t} \operatorname{Tr}\left(A_{l}\right)^{m}
$$

that we have just reproven To those already familiar with their formula it will be clear that all we have done is to make evident the inverse system of finite complexes $K_{a b}$ whose ghost haunted their purely combinatorial arguments

Remark 2 The transformation $A_{0}=L_{0}^{*} R_{0 *}$ is just the transition matrix of the transition relation $A$ For large $m$ it makes the dominant contribution to the alternating sum and one can view the other terms as higher dimensional corrections 
Remark 3 Suppose $I$ is transitive Then the equivalence classes form a set $\mathscr{P} / I$ and $\Omega$ embeds in the shift $(\mathscr{S} / I)^{\mathbb{Z}}$ and so is sofic Conversely every sofic $\Omega$ can be presented with $I$ transitive Then the matrix $A$ decomposes as a sum of blocks $B_{j k}$, $J, k \in \mathscr{Y} / I$ The exterior powers $\Lambda^{\prime} B_{j k}$ of these blocks form a block decomposition of $A_{i}$ and so

$$
\zeta_{f}(t)=\prod_{i \geq 0} \operatorname{det}\left(I-t\left(\Lambda^{t} B_{j k}\right)\right)^{(-1)^{t+1}}
$$

This is a readily computable formula for the zeta function of a sofic system

Remark 4 When $\pi$ has bounded fibers, a theorem of Hurewicz [H] implies that $\Omega$ has finite dimension In particular this holds for FP $\Omega$ In fact one only needs $\Omega$ expansive [Ma] A more direct proof for FP systems can be found in the exercises in [R, Chap 7], and it also follows from the fact that the dimension of the complexes in the inverse system $K_{a b}$ are bounded We conjecture that the metric $d$ of Lemma 2 has finite Hausdorff dimension

Remark 5 We mention a simple formula for the cohomology of a finite complex $K$ Form the exterior ring $E K^{0}$ with generators the vertices $v$ of $K$ and the relations $v^{2}=v v^{\prime}+v^{\prime} v=0$ Thus $E K^{0}$ is a free abelian group of rank $2^{\left|K^{0}\right|}$ Let $\mathscr{I} \subset E K^{0}$ be the span of the products of nonincident vertices, $1 \mathrm{e}$ sets of vertices that do not span a simplex Clearly $\mathscr{I}$ is an ideal in $E K^{0}$, which we call the nonincidence ideal The quotient ring $E K^{0} / \mathscr{I}$ is the cochain complex of $K$ with $\mathbb{Z}$ coefficients The differential $d$ is multiplication by the vertex $\operatorname{sum} \sum_{v \in K^{0}} v$

For a complex such as $F_{a b}, K_{a b}$ defined by filling in a graph, the nonincidence 1deal $\mathscr{I}$ is generated by quadratic relations $v v^{\prime}=0$ for $v, v^{\prime}$ not joined by an edge This gives a simple presentation of the cochain complexes used in this section

\section{REFERENCES}

[BI] P Blanchard Symbols for cubics and other polynomials To appear in Trans Amer Math Soc

[B1] R Bowen Equilibrium States and the Ergodic Theory of Anosov Diffeomorphisms Sprınger Lecture Notes in Maths 4701975

[B2] R Bowen On Axiom A Diffeomorphisms CBMS Reg Cónf 35, A M S, Providence, 1978

[BH] B Branner \& J Hubbard The iteration of cubic polynomials Preprint

[CP] E Coven \& M Paul Sofic systems Israel J Math 20 (1975) 165-177

[DGS] M Denker, C Grillenberger \& K Sigmund Ergodıc Theory on Compact Spaces Springer Lecture Notes in Maths 5271976

[ES] S Ellenberg \& N Steenrod Foundations of Algebraic Topology Princeton U P, 1952

[FLP] A Fath1, F Laudenbach \& V Poenaru Travaux de Thurston sur les surfaces Asterisque 66-67 1979

[F1] D Fried Zeta functions of Ruelle and Selberg, I Ann Sc1 ENS 19 (1986), 491-517

[F2] D Fried Rationality for isolated expansive sets Advances in Math 65 (1987), 35-38

[F3] D Fried Natural metrics on Smale spaces C R A S 297 (1983) 77-79

[G1] J Guckenheimer Axıom A and no cycles imply $\zeta_{f}(t)$ rational Bull Amer Math Soc 76 (1970) 592-594

[G2] J Guckenheimer Endomorphisms of the Riemann sphere Proc Symp Pure Math 14 A M S, Providence, 1970, 95-123

[H] W Hurewicz Uber dimensionerhohende stetige Abbildungen J fur Math 169 (1933) 71-78

[K] J Kelley General Topology Van Nostrand, 1955

[Kr] W Krieger On sofic systems I Israel J Math 48 (1984) 305-330 
[Ma] R Mañe Expansıve homeomorphisms and topological dimensıon Trans Amer Math Soc 252 (1979) 313-319

[M] A Manning Axıom A diffeomorphisms have ratıond zeta functions Bull London Math Soc 3 (1971) 215-220

[R] D Ruelle Thermodvnamic Formalism Addison Wesley, Reading, 1978

[We] B Weiss Subshifts of finite type and sofic systems Monatsh Math 77 (1973), 462-478 